\title{
Evaluation of Failure of Oestrus Synchronization and Mass Insemination in Conception Rate of Dairy Cows in Silte Zone
}

sharew mekonnen Haile ( $\square$ sharewmekonnen@gmail.com )

Werabe University

Tigist Wondala

werabe university

Mesobework kassa

Werabe university

Research Article

Keywords: failure, oestrus synchronization, mass insemination

Posted Date: July 19th, 2021

DOI: https://doi.org/10.21203/rs.3.rs-638206/v1

License: (c) (i) This work is licensed under a Creative Commons Attribution 4.0 International License.

Read Full License 


\section{Abstract}

The objective of this study was to evaluate failure of oestrus synchronization and mass insemination in the conception of dairy cattle in Silte zone. To evaluate the mechanism of the failure of OSMI conception rate we have collected (HF, jersey, and HF×Boran breed semen) from the three districts and evaluated their quality in NAGII laboratory thereafter bought 1000 qualified HF semen straw and distributed in the 3 districts. 150 cows were brought by farmers for services, and from those cows 130 cows were fulfil the precondition for this program and injected them with $2 \mathrm{ml}$ of PGF2 alpha hormone. Among the 130 injected cows, $72(55.38 \%)$ of them were responded from day 2 up to day 3 after hormone treated and then inseminated them. The lower percentages of conception rate which was observed in this study were associated with low number of AIT and mass number of cows provided during OSMI program, lack of awareness about heat detection, lack of inseminator equipment and cows did not properly managed after hormone and semen administered as we evaluated. As a result, the quality of semen in Dalocha and Sankura districts was facing with quality problem. We have checked the AITs efficiency through placing six cow per AIT thereafter they inseminated them. As a result, there was no AIT efficiency difference on conception rate based on their working experience in this study. Generally, there is a great need of skilled and experienced technician and capacity building of farmers in heat detection, husbandry practices, and good handling practice of semen in the office and improvement strategy. Therefore, improvement in facilities and management should be necessary before implementing an effective estrous synchronization and mass artificial insemination programs.

\section{Introduction}

In Ethiopia, studies pertaining on hormone- assisted oestrus synchronization in cattle started in the late 1980 s by a team of researchers in the animal health and reproduction section of the International Livestock Center for Africa (ILCA), and International Livestock Research Institute (ILRI). The research program was initiated with a long-term objective of integrating emerging reproductive technologies, such as estrus synchronization and mass insemination, Al, embryo transfer and associated techniques to improve the genetics and breeding of indigenous cattle for desirable traits and to explore the opportunities of using these technologies for genetic conservation of indigenous cattle in Africa. This focus of the research program was broadly defined as genetic improvement of cattle for milk and meat production, genetic improvement of trypanotolerant cattle breeds and genetic improvement of cattle for feed utilization efficiency (Azage et al., 2016).

To improve the efficiency of artificial insemination practices in Ethiopia, hormonal synchronizations of oestrus has been available for the past few years and has enjoyed success as a tool to make artificial insemination more practical (Azage et al., 2012; Gizaw et al., 2016). However, farmers expressed low satisfaction with the service, although evaluation of the technology by farmers is confounded with low conception rates (Gizaw et al., 2016). The development of effective methods of estrus synchronizing and ovulation has been

based on our understanding of the physiological and hormonal mechanisms controlling the 
estrous cycle and the initiation of estrous cyclicity and pre -puberty in heifers and postpartum cows. Estrus synchronization products (PGF2a, MGA, CIDR, and GnRH) and protocols have changed over time, the basic physiological principles underlying how these products work have not. An understanding of how these products impact the bovine estrous cycle and an understanding of how management decisions impact pregnancy success will affect on the success of any reproductive program. Estrus synchronization and artificial insemination (Al) are among the most powerful and applicable technologies for genetic improvement of beef herds (Seidel, 1995). For a synchronization program to be successful, herd nutrition (cattle must be in good body condition), cycles of estrus (must be a normal cycle), and herd health (free from diseases and parasites) and weight of the animals must be considered (Lamb, 2010). Moreover, Azage et al. (2012) reported that awareness creation, proper training, careful animal selection (good body condition score, cows free from disease and with functional ovaries), good animal handling facilities at a convenient location, a well-trained, organized and motivated multidisciplinary team (livestock science, feed and nutrition experts, veterinarians, Al technicians, etc.) actively participant community and proper leadership and coordination are key elements for the success of synchronization program. The change of hormone- treated dairy cows was not as expected in Ethiopia (Gizaw et al., 2016). This is due to

- Lack of proper understanding of the concept of oestrus synchronization by woreda experts and farmers.

- The communication of farmers with woreda experts in explaining the concept and process involved in oestrus synchronization was very low and the selection of unsuitable animals

- Poor preparation of materials for oestrus synchronization and Al service like shortage of liquid nitrogen and semen delayed the implementation plan.

- Transport and fuel shortage limited the movement of experts to supervise field activities.

- Weak support from administrative bodies and assignment of woreda experts to other activities after the synchronization work.

- Poor oestrus detection by farmers and exposure of hormone- treated cows to local bulls.

Cattle breeding are mostly uncontrolled in Ethiopia making genetic improvement difficult (Azage et al., 1995). Low pregnancy rate following artificial insemination in most African countries is attributed to poor semen quality, poor semen handling procedures, inadequate insemination skills, poor estrus detection, and wrong time of insemination (Azage et al., 1995).

\subsection{Statement of the Problem}

According to the cited by Desalegn (2008) in Tgriy region, estrus synchronization and mass insemination and conception rate were extremely low performance mainly due to the lack of skilled artificial insemination technicians, using of fixed time insemination in the synchronization protocol, unplanned strategic feed supplementation of synchronized cattle and animal selection problems. In Southern nation, nationality and people of region, the conception rate using synchronization hormone was $33.3 \%$ 
(Desalegn, 2008). Starting with 2012 up to 2020, records indicated that in Silte zone many cows were synchronized and inseminated. However, they faced for 7 year's trial a failure of conception rate after synchronized and mass insemination and so far no detailed comprehensive evaluation and assessment to analysis about the cause of failure of oestrus synchronization and mass artificial insemination in conception rate thereafter there were no detailed general study pertaining to the results and status of this program which addresses Silte zone bureau of livestock and fishery resource (BoLFR 20122020). Therefore, this study was designed to evaluate the cause of, failure of oestrus synchronization and mass artificial insemination in conception rate using PGF2 alpha treatment \& identify the problems why they faced such kind of failure problems associated with this technology in Silte zone, the southern nation nationality people of Ethiopia.

\subsection{General Objective}

- The overall objective of this research is to evaluate failure of oestrus synchronization and mass insemination in the conception rate of dairy cattle in the silte zone.

\subsubsection{Specific Objectives}

- To determine semen quality by estrus response per breed and districts in the study area.

- To compare the efficiency of Al technicians on conception rate based on working experience

- To evaluate semen quality by laboratory test in NAGIl laboratory.

\section{Materials And Methods}

\subsection{Description of the Study Area}

The study was conducted from October 2019 to April 2020 in Silte zone. Silte zone is one of the fourteen zones in the Southern Nation Nationality and People Regional State (SNNPRS). The Zone is bordered on the south by Alaba special woreda and the south west by Hadiya, East by the Oromia region, on the north by Gurage zone. The zone's main town (Worabe) is located on the road to Hosanna, just $172 \mathrm{~km}$ from Addis Ababa and it has 10 districts and 3 administrative town. Silte zone has different agro-ecological zones ranging in altitude between 1500 and 3700 masl; namely, highland (Dega) representing about $20.5 \%$ and midland (Woynadega) comprising $79.5 \%$ of the zone. The average temperature ranged from 12 - $26 \mathrm{OC}$ and the average annual rainfall ranges from $780-1818 \mathrm{~mm}$, Zonal bureau of agriculture (ZBOA 2010).

Different types of livestock populations are found in the area. From the total of 10,543,129 livestock population found in the region, $17 \%(1,793,033)$ are found in Silte zone. Out of this, $29.3 \%(525,178)$ are cattle of which $99.5 \%$ are local breeds with the remaining $0.5 \%(2,556)$ representing cross breeds (ZBOA 2010). Cattle are used as a source of draft power, manure, milk, meat, and a source of income to the sell of animals. The main livestock feed sources in the area are natural pastures and crop byproducts including hay and teff and wheat straw zonal bureau of agriculture (ZBOA 2010). 


\subsection{Sample Size Determination}

From Silte zone, three districts (Dalocha, Lanfro, and Sankura) were selected using multistage stratified purposive sampling technique based on potentiality of the districts and proximity to animal handling crush and cattle population, number of availability of synchronization and, Al practice, attitude of farmers to adopt oestrus synchronization and mass insemination (OSMI) technology, accessibility, and availability of infrastructure. The number of farmers involved in the OSMI was identified from district record data.

\subsection{Design of experimental animals}

For convenience, the current study was carried out on a total of 130 local and crossbred cows selected purposively. 87 local and 43 crossbreds of cows were selected purposively from the 3 districts. Preconditions fulfilled by the selected cows for estrus synchronization before the practically started. Body condition score range from 2 to 5 on a scale of 1 to 5 . Rating the body condition was done subjectively based on fat cover and flesh over the rips, loin and tail head (Roche et.al. 2009). Prior to the start of the experiment, status of reproductive problem of cows was confirmed by artificial inseminator through rectum palpation. Animals that included to experiment they possess mature corpus luteum on either of ovaries.

\subsection{Laboratory experiment}

To identify the failure of conception rate, from Dalocha (23), Lanfro (33), and Sankura (37) straw semen were collected already distributed by zone livestock and fishery resource office. Thereafter, take to in National Animal Genetic Improvement Institute (NAGII) laboratory and checked motility and quality of semen appendix 2, figure 2).

\subsection{Synchronization protocol and practical procedures}

The protocol of synchronization was single pgf2 alpha protocol. Tested and qualified HF straw semen bought from NAGIl and used for insemination purpose in Lanfro and Dalocha Sankura districts. Training was given for AITs before the research was performed about how to palpate the presence of corpse lutetium $(\mathrm{CL})$ before the cow to be inject hormone to prevent abortion due to hormone treatment and ovarian dis functionality (appendix 2, figure 1). After a single injection of the PGF2a hormone, 130 animals were synchronized for a fixed time Al. The female's animals which were diagnosed to be cycling with the presence of functional CL was determined through rectal palpation by Al technician, were injected (2ml) PGF2a intramuscular. The protocol used for the experiment was single injection Out of 130 cows brought to the three districts, only from Dalocha (50), Lanfro (50) and Sankura (30) were selected purposively based on body condition, age, health status, and absence of pregnancy during synchronization. Finally pregnancy diagnosis was carried out at 4 months of post-artificial insemination by rectal palpation and recorded. The questionnaire was administered to the Al technicians which was focused on the factor affecting conception rate in OSMI program in the study areas. 


\subsection{Effects of AITs work experience on conception rate}

To determine the effects of AITs work experience on conception rate artificial insemination technicians (AITs), were classified in to three groups thereafter they inseminated six cows per each AITs based on their working experience using already selected cows in the three districts using the same semen and environment.

\subsection{Collected Data}

Breed type, semen, number of hormone treatment responded cow, conception rate, factor affecting conception rate in OSMI program from 9 AIT and 4 livestock expert were collected.

\subsection{Data analysis}

The data were interred in Microsoft Excel and SPSS, checked, and analyzed by descriptive statistics using SPSS and SAS computer software program (versions 20 and 9,) respectively. Quantitative data obtained from the experiment was analyzed by using one-way ANOVA. Whereas qualitative data analyzed by chisquare using cross tabulation.

The Model used for conception rate in OSMI program of dairy cows

\begin{tabular}{l} 
Where Yij=Observed values of semen quality(motility, viability) on conception rate in \\
$\begin{array}{l}\text { OSMI program) } \\
\mu=\text { Overall mean } \\
\text { ai=Random effect of } i^{\text {th }} \quad \text { districts ( } i=3 \text { : Dalocha , Lanfro and Sankura) } \\
\text { ei=residual error }\end{array}$ \\
\hline
\end{tabular}

The Model used for conception rate in OSMI program of dairy cows for bull breed effect

$$
\text { Yij }=\text { p+bj +ej }
$$

Where $\mathrm{Yi}=$ Observed values of semen quality(motility, viability) on conception rate in OSMI program)

$\mu=$ Overall mean

$b j=$ fixed effect of $j$ th sire breed blood level $(j=3: 50 \% H F * B O, 100 \%$ HF exotic breed and Jersey)

ej $=$ residual error

Conception and oestrus response rate determination

conception rate $\mathrm{CR}(\%)=\frac{\text { Numberof conceivedcows } / \text { heifers }}{\text { number of inseminatcows } / \text { heifers }} * 100$

Oestrus rate $\mathrm{OR}(\%)=\frac{\text { Numberof treated cows/heifers }}{\text { Number of responsed cows } / \text { heifers }} * 100$ 


\section{Results}

\subsection{Evaluation of oestrus response and conception rate}

The results pertaining to the oestrus response and conception rates using single injection PGF2 alpha are presented in Table 1. The study shows that most of the hormone treated cows show responsive. About $44.6 \%$ of cows were not response after PGF2 $\alpha$ treated. Furthermore, the conception and oestrus rate were significantly different across the study areas of Dalocha (D), Lanfro (L), and Sankura (S). The studies further indicate that most of the inseminated cows were not conceived. The results pertaining to that among130 numbers of inseminated cows were 72 of them responsive. Whereas the remaining cows did not show heat, signs as a result they were not inseminated as shown in Table 1.

Table 1. The overall conception and response rate of cows in Silte zone per district.

\begin{tabular}{lcccccccc}
\hline & \multicolumn{7}{c}{ Districts } \\
\cline { 2 - 9 } & Dalocha & \multicolumn{2}{c}{ Lanfro } & Sankura & Overall \\
\hline Description & $\mathrm{N}$ & $\%$ & $\mathrm{~N}$ & $\%$ & $\mathrm{~N}$ & $\%$ & \\
\hline Number of hormone treated cows & 50 & 100 & 50 & 100 & 30 & 100 & 100 \\
Number of responsive cow (OR) & 25 & 50 & 30 & 60 & 17 & 56.67 & 55.4 \\
Number of inseminated cow & 25 & 100 & 30 & 100 & 17 & 100 & 100 \\
Number_conceived cow (CR) & 9 & 36 & 12 & 40 & 2 & 11.76 & 29.3 \\
\hline
\end{tabular}

OR= Oestrus rate, $C R=$ conception rate, $N=$ number of cattle

\subsection{The effect of breed on estrus response and CR}

The findings as presented in Table 2 shows that there were significance differences in oestrus response and conception rate between native and cross cow breeds across the studied locations. The results further indicate that the percentage of conception rate between breeds was too varied $(p<0.001)$, i.e., cross cow which was higher $(p<0.001)$ in CR.

Table 2. The effect of single dose PGF2 $\alpha$ on estrus response and conception rate per breed

\begin{tabular}{llllrl}
\hline $\begin{array}{l}\text { Breed } \\
\text { type }\end{array}$ & $\mathrm{N}$ & $\begin{array}{l}\text { PGF2 } \alpha \text { treated } \\
\text { cows }\end{array}$ & $\begin{array}{l}\text { Responsive } \\
\text { cow(OR) }\end{array}$ & $\begin{array}{l}\text { Inseminated } \\
\text { cow }\end{array}$ & $\begin{array}{l}\text { Conceived } \\
\text { cow(CR) }\end{array}$ \\
\hline Native & 87 & 87 & $40(45.97)$ & $40(45.97$ & $9(22.5)$ \\
Cross & 43 & 43 & $32(74.42)$ & $32(74.42)$ & $14(43.75)$ \\
Overall & 130 & 130 & $72(55.38)$ & $72(55.38)$ & $23(31.94)$ \\
\hline
\end{tabular}


The number outside the the bracket is represented: number of cows and inside the bracket is represented percent of $\mathrm{OR}$ and $\mathrm{CR}, \mathrm{N}=$ total number of animals, $\mathrm{OR}=0$ estrus response, and $\mathrm{CR}=$ conception rate

\subsection{Effect of district and breeds on semen quality}

\subsubsection{Effect of districts on semen quality}

The results indicated that the quality of semen after laboratory results was presented in Table 3, per district. As indicated by Table 3, there was a significance difference between districts in semen quality. Depend on motility, Dalocha district was lower as compared to the remaining two districts as indicated in (Appendix1).

Table 3. Analysis of variance table for semen motility affected by districts

\begin{tabular}{lll}
\hline Source of variation & Degrees of freedom & mean square \\
\hline Rep & 37 & $157.27^{\text {NS }}$ \\
District & 2 & $1132.28^{* *}$ \\
Error & 54 & 9368.77 \\
\hline Total & 93 & \\
\hline
\end{tabular}

NS $=$ non-significant, $* *=$ significant at $\mathrm{p} \leq 0.01$

\subsubsection{Effect of breed on semen quality}

The current result shows that the quality of semen was significantly difference per breed. As indicated by Table 4, Jersey breed semen was lower motility as compared to Holstein Frisian and Holstein Frisian cross with Borena breed semen motility. Depend on semen motility of HF*Bo breed, was higher motility than Jersey and Holstein Frisian breeds as indicated in (Appendix1).

Table 4. Analysis of variance table for motility affected by breed

\begin{tabular}{lll}
\hline Source of variation & Degrees of freedom & mean square \\
\hline Rep & 37 & $157.27 N S$ \\
Breed & 2 & $1132.28^{* *}$ \\
Error & 54 & 9368.77 \\
\hline Total & 93 & \\
\hline
\end{tabular}

$\mathrm{NS}=$ non-significant, $* *=$ significant at $\mathrm{p} \leq 0.01$

\subsection{Overall summary of semen quality evaluation based on motility standard per district}

The result was shown that the summary of semen quality presented in Table 5 the overall percentage of semen quality (85.7\%) of semen sample was pure and Standard and above the standard motility quality on the other hand $14.3 \%$ was below the standard semen 
motility quality as identified in NAGII of laboratory (in appendix 2 figure 2). Semen quality was a significance difference $(\mathrm{p}<0.05)$ across in the study districts. The standard and above the standard motility of bull semen was (65.22\%) in Dalocha (100\%) in Lanfro compared to Sankura (89.19\%) districts in the study area.

Table 5. The overall summary of semen quality evaluation laboratory result per districts

\begin{tabular}{llllllll}
\hline & \multicolumn{7}{c}{ Districts } \\
\cline { 2 - 7 } & Dalocha & \multicolumn{2}{c}{ Lanfro } & \multicolumn{2}{l}{ Sankura } & Overall \\
\cline { 2 - 7 } Semen quality\% & $\mathbf{N}$ & $\%$ & $\mathbf{N}$ & $\%$ & $\mathbf{N}$ & $\%$ & $\%$ \\
\hline Standard and above the standard motility & 15 & 65.22 & 33 & 100 & 33 & 89.19 & 85.7 \\
Below the standard motility & 8 & 34.78 & 0 & 0 & 4 & 10.81 & 14.3 \\
Total & 23 & 100 & 33 & 100 & 37 & 100 & 100 \\
\hline
\end{tabular}

$N=$ number of straw semen

\subsection{Cause of failure in conception rate in synchronization and mass insemination program}

The findings in table 6 further indicated that, low number of of AIT and mass number of cows provide during OSMI program, lack of awareness about heat detection of farmers and cows did not properly managed after hormone and semen administrated were ranked 1-3 those indicate that the major limiting factors across the study areas reported by artificial inseminator and livestock expert. Figure 3 in appendix 2 show that over stressed during OSMI program this is the evidence listed below table.

Table 6. Ranks of 9 AITS and 4 livestock expert response on cause of failure of conception rate in OSMI program in the study areas

\begin{tabular}{|c|c|c|c|c|c|c|c|c|}
\hline \multirow[b]{2}{*}{ irameters } & \multicolumn{8}{|c|}{$\begin{array}{l}\text { Ranks of cause of failure of conception rate in } \\
\text { OSMI program }\end{array}$} \\
\hline & 1 & 2 & 3 & 4 & 5 & 6 & index & Rank \\
\hline $\begin{array}{l}\text { JW no of AIT and mass number of } \\
\text { w present during OSMI }\end{array}$ & 47 & 41.1 & 1.9 & 10 & 0 & 0 & 0.25 & $1^{* *}$ \\
\hline $\begin{array}{l}\text { ick of awareness about heat } \\
\text { tection of farmers }\end{array}$ & 10 & 21.8 & 38 & 23.7 & 3 & 3.5 & 0.194 & $3 * *$ \\
\hline $\begin{array}{l}\text { Jws not properly managed after } \\
\text { irmone and semen treated }\end{array}$ & 39 & 14.5 & 35.9 & 10.3 & 0 & 0 & 0.229 & $2 * *$ \\
\hline w feeding management practice & 3 & 15 & 1.48 & 56 & 24.2 & 0 & 0.15 & 4 \\
\hline men quality & 0.3 & 5.8 & 12.7 & 0 & 33.8 & 47.4 & 0.094 & 5 \\
\hline ick of inseminator equipment & 0.7 & 1.7 & 10 & 0 & 38.8 & 48.8 & 0.085 & 6 \\
\hline
\end{tabular}

$* *=$ double stars indicates that more challenging problem

\subsection{Effect of AITs working experience on CR in OSMI program}


The result indicated in the table 7 pertained that the effect of work experience of artificial insemination technician (AIT) was statistically significantly difference across the three AITs based on working experience in Sankura district. Based on their working experience, they inseminated individually 6 cows per AITs. As a result, AIT1 from 6 inseminated, cows no one cows were conceived (PD+), but he has 4 years' experience in Sankura district. This show that there were AITs efficiency difference rather than working experience.

Table 7. Effect of AITs working experience on CR in OSMI program in Sankura districts

\section{Sankura district}

\begin{tabular}{lccc}
\hline Jame of AITs & Work Experience of AIT per year & PD+ (\%) & PD-(\%) \\
\hline IT1 & 4 & 0 & $6(100)$ \\
IT2 & 2.3 & $2(33.3)$ & $4(66.7)$ \\
IT3 & 5 & 0 & $6(100)$ \\
\hline
\end{tabular}

$P D+=$ pregnancy positive and $P D-=$ pregnancy negative

The result showed in the table 8 concerned that the effect of work experience of AITs on conception rate was statistically significantly difference between AITs 1 and2 in Dalocha district. In same way AITs 2 differ with AITs3 on conception rate in Dalocha district. AIT1 and AIT3 have better performance as compared to AIT2 but their working experience were differed as a result the conception rate was not based on working experience in Sankura district. AIT3 has 2 year experience from the total inseminated cows two cows were conceived $\left(\mathrm{pd}^{+}\right)$, but he has lower working experience as compared AIT1 and AIT2.

Table 8. Effect of AITs working experience on CR in OSMI program in Dalocha districts

$$
\text { Dalocha }
$$

\begin{tabular}{|c|c|c|c|}
\hline Jame of AITs & Work Experience of AIT per year & $\mathrm{PD}+(\%)$ & PD-(\%) \\
\hline IT1 & 6 & $4(66.67)$ & $2(33.33)$ \\
\hline IT2 & 3 & $1(16.67)$ & $5(83.33)$ \\
\hline IT3 & 2 & $4(66.67)$ & $2(33.33)$ \\
\hline
\end{tabular}

The results reported in Table 9 show a dependence of the conception rate on inseminator working experience related factors as well as those of the efficiency of them. Indeed, the conception rate varies significantly by AITs $(\mathrm{p}<0.02)$ in Lanfro district. 
Table 9. Effect of AITs working experience on CR in OSMI program in Lanfro districts

\begin{tabular}{|c|c|c|c|c|}
\hline \multicolumn{5}{|c|}{ Lanfro district } \\
\hline Jame of AIT & Work & Experience of AIT per year & $\mathrm{PD}+(\%)$ & PD-(\%) \\
\hline IT1 & 2.5 & & $3(50)$ & $3(50)$ \\
\hline IT2 & 5 & & $4(66.67$ & $2(33.33)$ \\
\hline IT3 & 7 & & $5(83.33)$ & $(16.67)$ \\
\hline
\end{tabular}

\section{Discussion}

\subsection{Hormonal response and conception rate}

The current finding, using a single injection of prostaglandin F2a protocol response rate was lower compared to the cited by Tegegn and Zelalem " Azage et al., (2012) who reported that $97.7 \%$ in HawassaDale milk shade and $100 \%$ in Adigrat-Mekelle milk shade areas. Adebabay et al., (2013) reported an oestrus rate was $89.3 \%$ in Bahir Dar milk shed; $72.3 \%$ oestrus rates reported in West Shoa zone in Bainesagn (2015) and Girmay et al., (2015) stated that 92.17\% in Wukro Kilte Awulaelo district, in Northern Ethiopia. Moreover, using the same protocol with the current study, 55.4\% oestrus rate was reported in eastern zone of Tigray region, Ethiopia (Tadesse, 2015). This difference may be due to the current research performed under small holder farmer management system and nutritional status of cows per partum after oestrus synchronization influenced the conception rate and postpartum ovarian cycles reported by (Wildeus, S. 2000). The difference may be due to animals reared in the intensive system showing higher pregnancy than extensively kept animals $13(62 \%)$ and 40 (55.6\%), respectively, as reported by Yeshimebet et al., (2017) and in addition, Son et al., (2007) reported that lower conception rate resulted from various factors related to lactation status, postpartum interval, and herd nutrition and management.

The current study on conception rate (29.3\%) was higher than $13.7 \%$ reported by Adebabay et al., (2013) in Bahir Dar milk shed area. This finding was in close agreement with those of $32.17 \%$ was reported by (Girmay et al., 2015); in Wukro Kilte Awulaelo district and Nordin et al., (2004) that records $32 \%$ CR. On the other side this finding was contradicted with the finding of Azage et al., (2012) 57.7\% in Hawassa-Dale milk shade, and in Adigrat- Mekelle milk shade $61.7 \%$ of pregnant animals was reported by(Tadesse, 2015). According to the AITs response, constraints that associated with this lower rate of pregnancy in the study districts were related to semen quality, low number of AIT and mass, number of cows present during synchronization and mass Al, feeding management, inability of heat detection of farmers were some of the associated factors affecting conception rate.

\subsection{The effect of PGF2 alpha on estrus response and CR per breed and district}

The effect of prostaglandins on estrus response and conception rate per breed \& district was presented in Table 2. District showed a significant $(p<0.05)$ effect on CR. Both Dalocha and Lanfro districts showed 
higher CR than Sankura. This study was in agreement with the study of Destalem G (2015) in the central zone of Tigray. The difference per district might be the lack of awareness creation in Sankura about the estrus synchronization during the study period. Most of the farmers answered during the study that they provided noncling cows and not gave birth for a long period of time. In other case, the experience, commitment, and acceptance of the technology by the farmer might be attributed to the difference in conception rate in the study area.

The current finding pertained that the effect of PGF2a on estrus response rate per breed was too varied $(P<0.05)$, which means it is higher successful in cross- breed compared to native breeds.This is contradicted with the study of Debir (2016) that stated $87.9 \%$ native and $86.9 \%$ cross cows respond oestrus. However, there was no significance difference effect of prostaglandin on estrus response per district. The results further indicate that the percentage of conception rate between breeds which implied that $C R$ in cross cows, was higher $(P<0.05)$. The conception rate per district was higher $(P<0.05)$ at Lanfro and Dalocha compare Sankura district.

\subsection{Semen quality and factors affecting conception rate}

The results as presented in Table 3-5 indicated that total, of ninety three straws of semen of different bulls as that of Silte zone were taken and checked for qualification in NAGIl laboratory for assessing the reasons why the synchronization and mass Al conception rate was so low, semen quality complained by farmers and AITs. The results indicated that there was a significant difference $(p<0.05)$ in semen quality among the three districts including per breed. This finding was in line with (Gebregiorgis, 2016). However, a special case was observed whereby the average value of motility for semen obtained from Dalocha district was as low as $36.95 \%$. Similarly, the average motility of semen obtained from Sankura district was $42.27 \%$ low compared to Lanfro district. Sire breed, or genotype had effect $(P<0.05)$ on frozen semen motility, this is in agreement with the study of (Debire, 2016).

The overall results shown in Table 6 about $85.7 \%$ of semen straw was good quality while 14.3\% was under the quality standared. The reduction of semen quality may be, transportation system, not timely top up of liquid nitrogen, breed type and not regularly checking up in the study district. Engidawork B (2018) studied that the motility of the same semen after freezing at the same center was $58.7 \%$, on average $21.3 \%$ loss due to freezing. After transportation and further storage at the regional Al service center, the average motility of frozen semen was further reduced to $49.4 \%$ (9.3\% loss). The loss happened might be through differences in chilling and freezing environment, including the volume and temperature of liquid nitrogen storage and transportation of semen from NAIC to the regional Al service center. As a result, not only low semen quality,and the fact that affect synchronization and mass Al fail in conception rate but also there were other factors like low number of AIT and mass number of cows present during synchronization and mass Al, lack of awareness about heat detection of farmers and cows did not properly managed after hormone and semen intake reported by AITs and farmers in the study districts. A study conducted by FAO (2005) indicated that if there are $40 \%$ or more of semen moving actively forward after freezing and thawing, the quality is acceptable for artificial insemination, which is consistent with the current study. 


\subsection{Effect working experience of Al technicians on conception rate}

The finding showed that conception rate was not based on AIT, s working experience but there was significance difference regarding to AIT, s skills on conception rate, this is contradicted with the report of Yeshimebt et al., (2017) stated that there was great variation in terms of using different working places with the highest degree of conception success $76.9 \%$ for T3 followed by T4 (70\%), T1 (54.6\%) and T2 (27.3\%) in North Shoa zone. The difference may be due to the technological application strategies, system of AITs, genotype of cows and farmer management system of cows after inseminated. The current finding was inclose agreement with the finding of( Muoffok et al., 2019) who reported that Ins 1 ( 63.4), Ins 2 (19.5), (Ins 3) (6.7), Ins 4 (6.0) and Ins 5 ( 4.3) in percentage.

\section{Summary And Conclusion}

As pertaining to the results of this study, using single injection, prostaglandin PGF2alpha was relatively effective to synchronize cows.

The results further indicate that the percentage of conception rate between breeds using single PGF2 alpha hormone treatment was too varied $(P<0.05)$. Cross cow was higher $(P<0.05)$ in $C R$. The conception rate per district had no significance difference between Dalocha and Lanfro, but it is a significance difference between Sankura and the two districts which means there was higher $(P<0.05)$ in Lanfro and Dalocha district as compared to Sankura district.

The lower percentages of conception rate which was observed in this study were associated with heat detection problems of farmers, low number of AIT and mass, number of cows proveded during synchronization and mass Al, lack of awareness about heat detection of farmers, lack of inseminator equipment and cows did not properly managed hormone and semen intake reported by AITs.

We conclude that the current study results, shown that the cause of OSMI conception failure was not only semen quality but also the low number of AIT and mass number of cows provided during synchronization and mass Al, low feeding management practice and lack of awareness about heat detection of farmers were the major limiting factors. To improve the effectiveness of this technology, skilled and experienced technicians as well as capacity building of farmers in heat detection, qualified semen, and improved breed and husbandry practices were of major concern.

\section{Recommendation}

- To improve OSMI conception and heat response rate the program must be done in a small scale and increase through time rather than mass cows provide at a time.

- Training and creating awareness for farmers must be giving before the OSMI program take place. 
- Even if there were many challenging problem that cause of failure of OSMI in the study areas it is important to perform by community breeding program service.

- Skilled and experienced technicians as well as capacity building of farmers in heat detection, qualified semen, and improved breed and husbandry practices are the major concerns.

- It needs checked by other research cooperatively with Werabe University, zone livestock and fishery resource office and stakeholder together for better result by using double dose of PGF2alpha treatment.

\section{References}

Adebabay K, Getinet Z, Yeshw as F, Temesegen A, Azage T 2013. Prostaglandin (PGF2 a) based oestrus synchronization in postpartum local cow s and heifers in Bahir Dar milk shed. Int. J. Pharm. Med. Bio. Sci. 2(4):37-43

Azage Tegegne, Awet Estifanos, Asrat Tera and Dirk Hoekstra 2012. Technological Options and Approaches to Improve Smallholder Access to Desirable Animal Genetic Material for Dairy Development: IPMS (Improving Productivity and Market Success) Experience with Hormonal Oestrus Synchronization.

Azage Tegegne, Dirk Hoekstra, Berhanu Gebremedhin and Solomon Gizaw 2016. International Livestock Research Institute (ILRI) February. 2016

Azage Tegegne, Tesfu Kassa, Mukasa-Mugerw E.1995. Aspects of bull production with emphasis on cattle in Ethiopia. Sperm production, capacity and semen characteristics. In: Proceeding of the Third National Conference of Ethiopian Society of Animal production, pp. 83-99.

Bainesagn Worku, 2015. Assessment of breeding practices and evaluation of estrus synchronization and mass insemination technique in dairy Cattle in West Shoa Zone. MSc thesis, Haramaya University, Haramaya, Ethiopia.

Desalegn Gebremedhin, Bekana M, Azage Tegegne, Blihu B 2009. Status of Artificial Insemination Service in Ethiopia. A paper presented at the 17th Annual Conference of the Ethiopian Society of Animal Production (ESAP), held at the Head Quarters of the Ethiopian Institute of Agricultural Research (EIAR), Addis Ababa, Ethiopia, pp. 87-104.

Destalem Gebremichael 2015.Breeding practice and estrus synchronization evaluation of dairy cattle in central zone of Tigray, northern Ethiopia M.Sc. Thesis June, 2015

Gebregiorgis Asebir, Alemselam Birhanu and Tadesse Gugsa 2016. Status of artificial insemination in Tigray regional state,"constraints and acceptability under field condition journal of dairy, veterinary \& animal research volume 3 issue $3-2016$.

Girmay G, Berihu G, Bahlibi W 2015. The effect of one shot Prostaglandin on Estrus Synchronization of Local and Holstein Friesian Cattle in and around Wukro Kilte Aw ulaelo District, Northern Ethiopia. J. Bio. 
Agric. Health 5(7):99-105.

Gizaw, S, Tesfaye, Y, Mekuriaw, Z, Tadesse, M, Hoekstra, D, Gebremedhin, B and Tegegne, A 2016. Oestrus Synchronization for Accelerated Delivery of Improved Dairy Genetics In Ethiopia: Results From Action Research And Development Interventions. LIVES Working Paper 12. Nairobi, Kenya: International Livestock Research Institute (ILRI).

FAO (Food and Agriculture Organization) .2005. Improving Artificial Breeding of Cattle in Africa Guidelines and Recommendations.

Mouffok1, A Allouni1, L Semara1,2 and F Belkasmi1,3, 2019. Factors affecting the conception rate of artificial insemination in small cattle dairy farms in an Algerian semi-arid area department of Agriculture and Livestock Sciences, University Setif 1, Algeria april 2019

Lamb G. Cliff 2010. Estrus Synchronization protocols for cows. North Florida Research and Education Center, Marianna, Florida. Proceedings, Applied Reproductive Strategies in Beef Cattle January 28 29, 2010; San Antonio, TX

Nordin Y, Zaini N, and Wan Zahari W.M., 2004. Factors affecting conception rate in dairy cows under selected smallholder production system J. Trop. Agric. and Fd. Sc. 32(2): 219-227.

Seidel, G. E. 1995. Reproductive biotechnologies for profitable beef production. In: Proc. Beef Improvement Federation. : P 28 Sheridan, WY.

Son, D.S., Choe, C.Y., Cho, S.R., Choi, S.H., Kim, H.J., Hur, T.Y., Jung, Y.G., Kang, H.G. and Kim, I.H. 2007. A CIDR Based timed embryo transfer protocol increases the pregnancy rate of lactating repeat breeder dairy cows. J. Reprod. Dev. 53, 1313-1318.

Tadesse $\mathrm{G}$ 2015. Effects of prostaglandin administration frequency,artificial insemination timing and breed on fertility of dairy cow s and heifers in eastern zone, of Tigray region, Ethiopia. MSc thesis, Mekelle University, Ethiopia

Tegegn Fantahun and Zelalem Admasu 2017. Evaluation of oestrus synchronization and mass artificial insemination service of dairy cattle in Mizan Aman area, Bench Maji zone, South West Ethiopia Vol. 8(1), pp. 1-4, January 2017 DOI: 10.5897/IJLP2016.0338

Wildeus, S. 2000. Current Concepts in Synchronization of Oestrous: Sheep and Goats. Journal of Animal Science, 77, 1-14.

Yeshimebet Chanyalew Tesfaye Z, Hulunim G, Lina G, Getachew K, Chekol D, Amare B, Ayele A and Yishak Tsegaye 2017. Evaluation of Two Estrus Synchronization Protocols in Dairy Cattle at North Shoa Zone Ethiopia /Animal Production. 19(2):93-100, 2017 ISSN 1411-2027 


\section{Declarations}

Acknowledgments. The authors would like to thank Tigst Wondala and Mesobework kassa as their encouragement, insight, guidance, and professional expertise for the suggestions on the analysis method. I also gratefully acknowledge Worabe university financial supporting during the study period.

Authors' contributions Sharew Mekonnen perform done the whole part of the research.

Tigst Wondala and Mesobework kassa commented, edited and guidance of the research. All authors read and approved the final manuscript.

Funding This work was supported by Werabe university. But the budget was already settled and not applicable.

Availability of data and materials. The datasets used and analyzed during the current study are available from the corresponding author on reasonable request.

Ethics approval and consent to participate. Not applicable

Consent for publication. Not applicable.

Competing interests. The authors declare no conflicts of interest .

\section{Author details}

1 Sharew Mekonnen senior lecturer in Werabe University, college of agriculture, department of animal science, Specialization animal breeding and genetics

Email address (sharewmekonnen@gmail.com)

Tigst Wondala senior lecturer in Werabe University, college of agriculture, department of animal science, specialization Dairy science technology

Email address(tigistwondala@gmail.com)

Mesobework kassa) senior lecturer in Werabe University, college of agriculture, department of animal science, specialization: animal nutrition

Email address (mesobeworkkassa@gmail.com)

\section{Supplementary Files}

This is a list of supplementary files associated with this preprint. Click to download.

- Appendix.docx 